\title{
Hypoglycemic effects of cocoa (Theobroma cacao L.) autolysates
}

\begin{abstract}
Fat, alkaloid and polyphenol contents of two clones of cocoa (UIT1 and PBC 140) were removed and the remaining powder was autolyzed at $\mathrm{pH} 3.5$ and 5.2. Based on the results, autolysates of UIT produced at $\mathrm{pH} 3.5$ exhibited the highest ability to inhibit $\alpha$-amylase activity. However, no $\alpha$-glucosidase inhibition activity was observed under the conditions specified. Autolysates produced under $\mathrm{pH} 3.5$ caused the highest amount of insulin secretion. In streptozotocin-diabetic rats, all cocoa autolysates significantly decreased blood glucose at $4 \mathrm{~h}$. To assure that the results from the assays were not due to the polyphenols of cocoa autolysates qualitative and quantitative tests were applied. According to their results cocoa autolysates were found to be free from polyphenols. Analysis of amino acid composition revealed that cocoa autolysates were abundant in hydrophobic amino acids. It can be suggested that besides other compounds of cocoa, its peptides and amino acids could contribute to its health benefits.
\end{abstract}

Keyword: Cocoa autolysates; Digestive enzymes inhibition; Insulin secretion; Hypoglycemic effect 